\title{
Visual and tactual form discrimination: Psychophysical comparison within and between modalities'
}

\author{
DEAN H. OWEN, ${ }^{2}$ OHIO STATE UNIVERSITY \\ AND DONAID R. BROWN, PURDUE UNIVERSITY
}

\begin{abstract}
Intermodal perceptual equinalence was invertiguted, wing viund and theturl oddity discriminetion and cross-modal matchist to-somple todes Four growps of Ss were presented with 100 problems made up of nendomby derived forms from five levels of sidedhess The sume quodratic relationulip was observed for all tosits as a function of complexity, with optinal peformmone ot eight sides Common information utifization, as indicated by interconelations of performance viables and by correlational analyses with differences in form mensures, was not as pronounced within side clusses. Evidence conceming the mature of pattem-feature unge in discrimination wos obtained from Eraphical analyses.
\end{abstract}

When a perceptual problem can be solved by exploring the stimulus objects vivually or tactually, or when part of the problem is presented via one modality and the remainder to the other modality, there is an opportunity to compare the information-handling characteristics of the two systems. In a situation employing multivarinte stimuli, some properties may be more salient for one modality than for the other, resulting in differential problem difficulty for the two modalities.

While Caviness and Gibson (1962) have demonstrated that tactual-to-visual matches can be made without previous experience, although performance improves with practice, most studies have been concerned with form discrimination and subsequent cross-modal transfer of training (Bjōtkman, Garvill, \& Molander, 1965; Caviness \& Gibson, 1964; Gaydos, 1956; Gibson, 1963; Lobb, 1965; Pick, 1965; Rudel \& Teuber, 1964). It has been shown that transfer does occur, sometimes assymetrically, but difficulty in interpreting and integrating the results of cross-modal studies seems to arise because there is no basis for determining why equivalence does or does not occur. Except for Pick (1965), the studies have suffered from a lack of stimulus quantification and hitile is known about the form dimensions along which discrimination, matching, and

Fig. 1. Example of a visual diveriaination oddity problem from the set at eight sidex. cross-modal transfer take place. Psychophysical knowledge of what stimulus information is used and how it is. used in transfer or cross-modal comparison should aid in understanding the processes involved.

In earlier studies, a comparison of the physical correlates of visual and tactual form complexity scales (Owen \& Brown, 1970) demonstrated a high degree of equivalence in information utilization. Other studies verified these findings using multidimensional scaling analyses (Brown \& Brumaghim, 1968) and an anchoring design (Brumaghim \& Brown, 1968). The present studies were concerned with investigating similar questions for pattem discrimination in the case of visual and tactual presentations.

\section{Subjects}

Two hundred male Purdue University undergraduates performed the visual-discrimination task and 96 performed the tactual-discrimination task. Ninety-six male Ohio State University undergraduates performed the two matching tasks, 48 in each condition. All Ss participated in fulfillment of an introductory psychology course requirement.

Pattern Selection and Experimental Design

Two hundred forms were sampled from the 1,000 forms generated by Brown and Owen (1967) according to the Method I rules for angular shapes of closed contours described by Attneave and Arnoult (1956). All forms were equated to an area of 1,250 square units and plotted in a $100 \times 100 \mathrm{~mm}$ coordinate system. For each problem, two forms from the same sidedness level were paired at random and one randomly designated as the "different" figure, providing 20 problems at each of five sidedness levels: $4,8,12,16$, and 20 sides. The tactual stimuli were constructed from very fine abrasive paper (Behr-Manning 360A), and glued onto a $100 \times 100 \mathrm{~mm}$ square of smooth poster board. The visual stimuli were transparencies, black on a white background, presented with the same area as the tactual forms on a rear-projection screen.

Problems were constructed for the four different conditions of presentation. The visual-discrimination (VVV) oddity problems consisted of five copies of the incorrect form and one copy of the correct or "different" form, a display used by Brown, Hitchcock, \& Michels (1962). The position of the "different" form was assigned randomly to one of the six possible positions for each problem, with the restriction that each position be used as nearly as possible an equal number of times at each sidedness level. Due to the extremely large number of slides required, no within-problem control of position effects was attempted. An example of a VVV problem from the set of eight-sided patterns is shown in Fig. 1. The three forms in the tactual-discrimination (TTT) oddity problems were arranged in a horizontal array, with the "different" form appearing an equal number of times in each position. Because the TTT task proved very time-consuming, Ss were divided into two groups of $48 \mathrm{Ss}$ each, with each $S$ receiving 50 problems, 10 from each side class.

Matching-to-sample problems were constructed for the two cross-modal
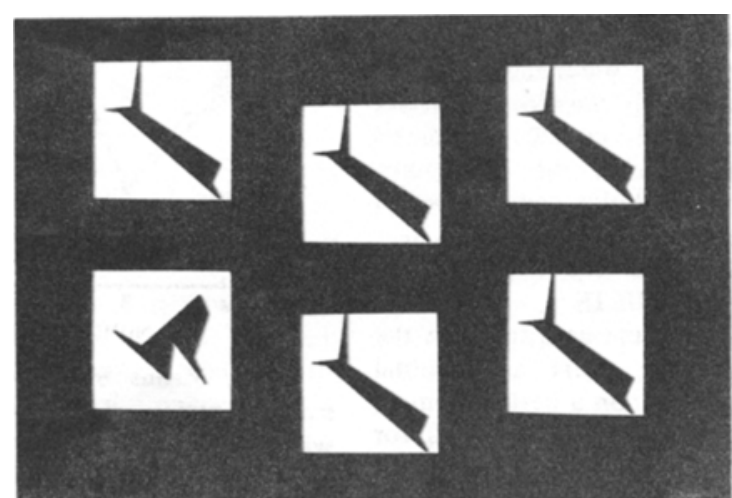

$(302$

Copyngh 1970, Psychonomic Joumals, Inc., Austin, Texas

Perception \& Psychophysies, 1970, Vol. 7 (5) 
situations since the task had been used by other researchers and seemed better suited to comparison of information from the two modalities. For visual-to-tactual matching (VVT) problems, two visual forms were presented above one tactual form, and for tactual-to-visual matching (TTV), one visual form was presented above two tactual forms, with position effects balanced. Order of presentation of problems for all tasks was randomized for each $S$ by a computer program with the restriction that each side class be represented in each consecutive block of five problems.

\section{Procedure}

In all tasks, Ss were tested individually with instructions to select the correct form as rapidly as possible without sacrificing accuracy. The $S$ initiated each trial by lifting his preferred hand from a plate, thereby starting a $1 / 100-\mathrm{sec}$ timer. His action also resulted in presentation of the rear-projected visual display in the VVV, VVT, and TTV takss and allowed him to begin exploring the tactual forms with his preferred hand in the VVT, TTV, and TTT tasks. The tactual forms were always explored successively from left to right in the TTV and TTT tasks, with the visual form(s) available for viewing during tactual exploration in the VVT and TTV tasks. For the VVV task, six lighted buttons were arranged in the same configuration as the forms shown in Fig. 1. When $S$ pushed the button corresponding to the position of the odd form, the timer stopped, while the display on the screen and the lights in the buttons were extinguished.

A curtain prevented $\mathbf{S}$ from seeing the tactual forms in the cross-modal matching experiments. In the VVT task, $S$ pushed a button below the visual form that matched the form he had explored tactually. Ss in the TTV task pressed down on the tactual form that matched the visual form projected above. A correct response stopped the timer and extinguished the visual display in both matching tasks.

The $S s$ in the TTT experiment were blindfolded and instructed to explore the three forms and press down on the odd form. If correct, his response stopped the timer and initiated a $1 / 2-\sec$ tone in his headset. A correction procedure was used in all tasks, i.e., $S$ responded until correct on each trial and practice problems preceded testing under all conditions. Latency to correct choice and number of errors were recorded on each trial.

\section{RESULTS}

Four techniques were used to assess the equivalence of the visual and tactual modalities and to obtain a better estimate of how pattern information is used for
Table 1

Tant Men

\begin{tabular}{r|rrrrrr}
\hline & \multicolumn{4}{c}{ Latencies in Seconds } & Errors \\
\cline { 2 - 5 } Sides & VVV & VVT & TTV & TTT & TTT \\
\hline 4 & 1.48 & 6.41 & 14.71 & 27.82 & 0.13 \\
8 & 1.22 & 6.03 & 13.56 & 29.93 & 0.11 \\
12 & 1.34 & 6.90 & 15.40 & 33.54 & 0.15 \\
16 & 1.40 & 7.64 & 18.48 & 36.53 & 0.18 \\
20 & 1.60 & $\mathbf{8 . 2 4}$ & 19.30 & 38.16 & 0.21 \\
\hline
\end{tabular}

discrimination: (a) Dependent-rariable comparisons were made by comparing task means across sidedness levels and by intercorrelating tasks; (b) differences in form measures were factor amalyzed and the factor scores were used in a multiple-regression solution to account for variation in performance; (c) differences in form measures, as well as area of nonoverlap of the two forms in a problem, were correlated with performance to assess the kinds of information that are most useful in discrimination; and (d) scattergraphs of dependent variables were plotted against form measures to reveal the relationship between performance and pattern characteristics.

\section{Dependent-Variable Comparioons}

Several points need clarification prior to consideration of the major analyses. First, analysis of variance of latencies for the six VVV correct positions indicated a significant variation in the position means (p<.01). Statistical correction was made by adjusting all problem means by the deviation of the correct position mean from the grand mean. Second, errors were negligible for all tasks except TTT, where an error rate of $16 \%$ was observed. Third,

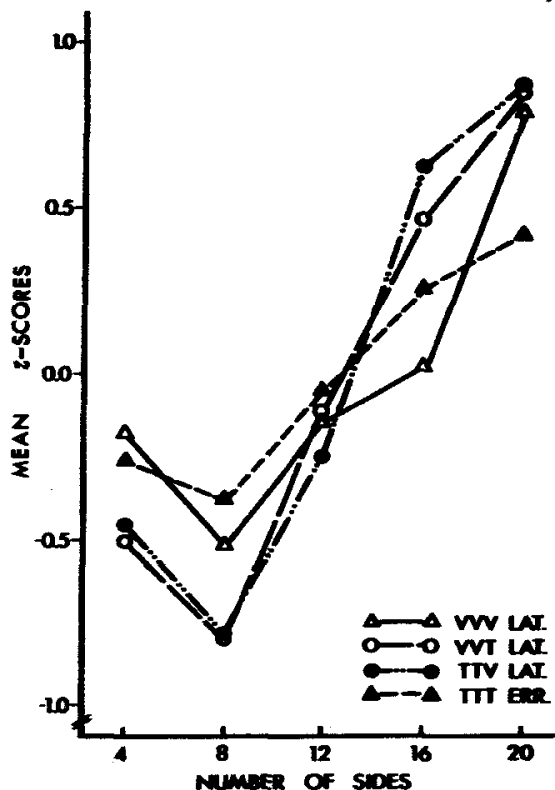

Fig. 2. Means by idednes level after standardination of the 100 problem scores within each tack. since it appears meaningful to compare task means that vary widely in magnitude, it is desirable for the purpose of illustration to present the relative difficulty of each sidedness level for each tak. Therefore, standard scores of the 100 problem scores in a task were computed and the mean standard score taken at each sidedness level.

VVV, VVT, and TTV latencies and TTT errors summarized across sidedness levels, shown in raw-score form in Table 1 and standard-scone form in Fig. 2, provided strong evidence for perceptual equivalence in problem-solving capability as a function of number of sides. Brown et al (1962) found the same pattern for VVV latencies as a function of sidedness. The TTT latencies, also shown in Table 1, with a linear component accounting for $98 \%$ of the variation among side clasees, provided the only exception to the general quadratic relationship.

Concerning the intercorrelations among the task, if common information was used in all four conditions then the coefficients hould be high and about equal. If, on the other hand, different stimulus relations were used in visual than in tactual discrimination, then cross-modal matching might make use of only those form attributes that were salient both visually and tactually. In that cane, the between-modality tasks should comelate more highly than the within-modality tasks.

The correlations among the tadks are sown in Table 2, computed for the total 100 problems from all five side classes and, as a within-class example, for the 20 problems at four sides. Two general statements can be made about the correlations. First, neither of the proposed altemative outcomes was observed. The corrchtions were neither consistently high nor consiatently ordered, although the tacks had some common variance. While part of the remnining variance may be due to unreliability of the dependent variables, that is not the case for the VVV latencies where splithalf reliability coefficients ranged from .91 at 20 sides to .99 at 4 sides. The second point has to do with the generally low correlations of the TTV

\begin{tabular}{|c|c|c|c|c|c|}
\hline \multirow[b]{2}{*}{ Tast } & \multicolumn{4}{|c|}{ Latencies } & \multirow{2}{*}{$\begin{array}{l}\text { Emor } \\
\text { TTT } \\
\end{array}$} \\
\hline & VvV & $\mathbf{V T}$ & $\mathbf{T T}$ & TTT & \\
\hline $\begin{array}{l}\text { VVV } \\
\text { VVT } \\
\text { TTV } \\
\text { TTTLat } \\
\text { TTIER. }\end{array}$ & $\begin{array}{l}-.69 \\
.38 \\
.69 \\
.65\end{array}$ & $\begin{array}{l}.62 \\
-57 \\
.82 \\
.70\end{array}$ & $\begin{array}{c}.44 \\
.69 \\
.68 \\
.66\end{array}$ & $\begin{array}{l}.59 \\
.71 \\
.61 \\
.93\end{array}$ & $\begin{array}{l}.47 \\
56 \\
.44 \\
.76 \\
--\end{array}$ \\
\hline
\end{tabular}


$T^{2}+x^{n}=3$

Conplutions of Factors (F) at Squared

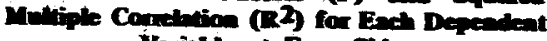
Variobse at Fowr spios

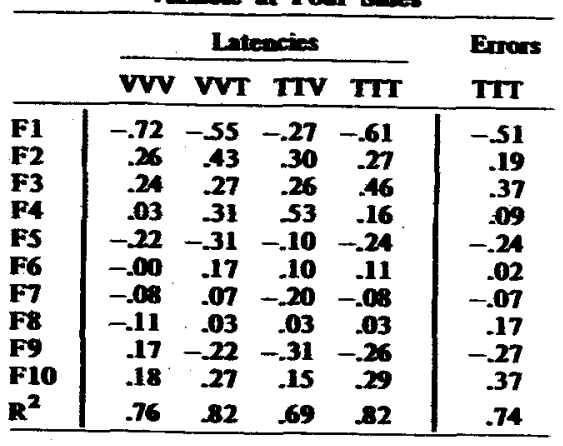

latencies with dependent variables and also with physical meanures and factor scores (see Tables 3 and 4). Considering the relatively high correlations of the VVT and TTT latencies, there is little reason to expect a problem with results of the TTV task, othe thon some factor of unrelinbility in the TTV data.

The correlations between the TTT latencies and errors were $.93, .90, .67, .63$, and 52 from 4 to 20 sides, showing a trend for the two varibles to become more uncorrelated as complexity increases. The trend was observed even though errors tend to increase with complexity and the correction procedure should inflate the correlation because errors result in longer latencies.

\section{Pyschophysioul Comperious}

Two approaches were taken in attempting to discover the stimulus information utitized in solving the problems. Principal-component factor andyses of the differences in $\mathbf{8 0}$ measures of the two forms in a problem were carried out within each side class. Enough factors were rotated to result in a high loading of at least one measure on each of as mmy factors as possible to allow interpretation of the factors. All 80 differences in measures were normalized by transformation via a normal probability table before factoriontion, since extremely large original scores from skewed distributions hxe an inordinate influence in the factor solution. Four sides is agrin chosen for the purpose of illustration, although it is neither the best nor the worst in terms of socounting for varintion in latencies. The factor scores representing the location of each problem along the 10 factor dimensions were used as predictors in multiple-regression solutions, resulting in the standardized beta-weights and percentages of variance accounted for shown in Table 3. Since the factors are independent, the correlation of each factor with a dependent variable is a beta-weight in the equation.
Examination of loadings of measures on factors indicated that Factor 1 is best described as representing the elongation of a form along an axis, independent of rotation. Factor 2 has high loadings from measures of angular variability, while Factor 3 is related to vertical extent of a figure. Length of perimeter has the highest londing on the fifth factor. If measures of the features useful in discrimination have high loadings on the first 10 factors, the beta-weights can be interpreted as an approximation to a perceptual strategy. Since a useful feature may load on two factors and not be represented in the regression equation, howrever, it is better to consider the squared multiple correlation as an index of how much variation in performance can be accounted for by the measurement system, as shown in the bottom row of Table 3 . The problems of locing useful measures not included in the first 10 factors and of inchuding dimensions not silient, of course, remain.

An estimate of which original measures were salient and the extent of their utilintion was made by correlating the dependent variables with differences in all $\mathbf{8 0}$ measures plus area of nonoveriap of the two figures in a problem. Area of nonoverlap was measured by a computer program that superimposes the matrices containing the two patterns that are specified by square units of area. Unit areas of each matrix that are enclosed by the form contain $1 \mathrm{~s}$ and areas that are not enclosed contain Os. Summing the two matrices results in $2 s$ in the area of overlap, equivalent to the translation overlap estimated optically by Boynton et al (1961). By scanning the matrix and changing all $2 s$ encountered to $0 s$, the area of nonoverlap can be computed by summing the remaining is. If perceptually useful, area of nonoveriap should be negatively correlated with latency.

Examples of the correlations are tabulated in Table 4. It must be noted that the measures sampled were correlated to various degrees, so that their contributions in accounting for performance were not independent. Two broad statements can be made that generalize across most measures: first, that the lowest correlations across side classes were usually at 12 sides, and second, the lowest correlations across tasks were usually for the TTV task. Nonoverlap tended to account for less variance in performance as complexity increased.

One of the most important findings in terms of understanding the perceptual processes involved came from the simplest level of psychophysical anslysis, that of graphical representation. Scattergraphs of latencies plotted against a measure having a respectable correlation with latency (e.g-, .60 or greater) typically reveal a triangular configuration as shown in Fig. 3. Short latencies were observed at any level of a salient attribute, while long latencies were observed with decreasing frequency as there was greater difference in amount of the property for the two forms in a problem. The three form pairs in Fig. 4 made up the problems represented by the numbered dots in Fig. 3. The forms in

Talle 4

Correlations of Selectied Measeres with Each Other and with the Denendent Varibles

\begin{tabular}{|c|c|c|c|c|c|c|c|c|}
\hline & \multicolumn{3}{|c|}{ Measures } & \multicolumn{4}{|c|}{ Latencies } & \multirow{2}{*}{$\begin{array}{l}\text { Emors } \\
\text { TTT }\end{array}$} \\
\hline & M1 & $\mathbf{M} 2$ & $\mathbf{M 3}$ & $\mathbf{v V}$ & $\mathbf{V} \mathbf{T}$ & TTV & $\mathbf{T T T}$ & \\
\hline & \multirow{5}{*}{$\rightarrow-$} & \multirow{5}{*}{$\begin{array}{l}.64 \\
--\end{array}$} & \multirow{5}{*}{$\begin{array}{l}.46 \\
55 \\
--\end{array}$} & \multicolumn{4}{|c|}{ At Four Sides } & \multirow{5}{*}{$\begin{array}{r}-.45 \\
-.46 \\
-.56\end{array}$} \\
\hline \multirow{4}{*}{$\begin{array}{r}\mathbf{M 1} \\
\mathbf{M} 2 \\
\mathbf{M} 3\end{array}$} & & & & -.50 & -.42 & -.33 & -.49 & \\
\hline & & & & -.68 & -.58 & -.35 & -.60 & \\
\hline & & & & -.64 & -.75 & -.55 & -.71 & \\
\hline & & & & \multicolumn{4}{|c|}{ At Eight Silos } & \\
\hline \multirow{3}{*}{$\begin{array}{l}M 1 \\
M 2 \\
M 3\end{array}$} & \multirow[t]{4}{*}{--} & \multirow[t]{3}{*}{.49} & \multirow{2}{*}{$\begin{array}{l}.34 \\
.77 \\
-\end{array}$} & -.32 & -.07 & -.22 & -.32 & \multirow{3}{*}{$\begin{array}{l}-.21 \\
-.32 \\
-.44\end{array}$} \\
\hline & & & & -55 & .00 & -.38 & -.45 & \\
\hline & & & -- & -54 & -.17 & -52 & -54 & \\
\hline \multirow{4}{*}{$\begin{array}{l}\mathbf{M} \\
\mathbf{M 2} \\
\mathbf{M}\end{array}$} & & \multirow{3}{*}{$\begin{array}{r}-.16 \\
--\end{array}$} & \multirow{3}{*}{$\begin{array}{r}-.45 \\
.19 \\
--\end{array}$} & \multicolumn{4}{|c|}{ At Twelve Sides } & \\
\hline & \multirow{3}{*}{--} & & & $\begin{array}{l}-.46 \\
-.27\end{array}$ & $\begin{array}{l}-.25 \\
-.09\end{array}$ & $\begin{array}{l}-.01 \\
-.02\end{array}$ & $\begin{array}{l}-.36 \\
-.07\end{array}$ & \multirow{2}{*}{$\begin{array}{r}-.31 \\
.23 \\
.08\end{array}$} \\
\hline & & & & .29 & -25 & .46 & .11 & \\
\hline & & & & & At $S$ & Sides & & \multirow{4}{*}{$\begin{array}{r}-.23 \\
.34 \\
-.27\end{array}$} \\
\hline \multirow{3}{*}{$\begin{array}{l}\mathbf{M 1} \\
\mathbf{M 2} \\
\mathbf{M 3}\end{array}$} & \multirow[t]{4}{*}{$-\cdots$} & \multirow[t]{3}{*}{.33} & \multirow{3}{*}{$\begin{array}{l}.05 \\
.46 \\
--\end{array}$} & -.23 & -.25 & .24 & .18 & \\
\hline & & & & -.47 & -.20 & .01 &.- .14 & \\
\hline & & & &.- .2 &.- .20 & -.38 & -.37 & \\
\hline $\mathbf{M}$ & & 31 & 07 & -75 & At iv & & & \\
\hline $\mathbf{M}$ & & - & .65 & -.49 & $\begin{array}{l}-.33 \\
-.49\end{array}$ & $\begin{array}{l}-.44 \\
-.13\end{array}$ & $\begin{array}{l}-.02 \\
-.02\end{array}$ & $\begin{array}{l}.04 \\
.23\end{array}$ \\
\hline $\mathbf{M 3}$ & & & - & -.23 & -.50 &.- .14 & -.20 & -.10 \\
\hline
\end{tabular}

MI Perimeter length

M2 Meximum second moment of perineter distribution

MS Arez of non-onerlop 


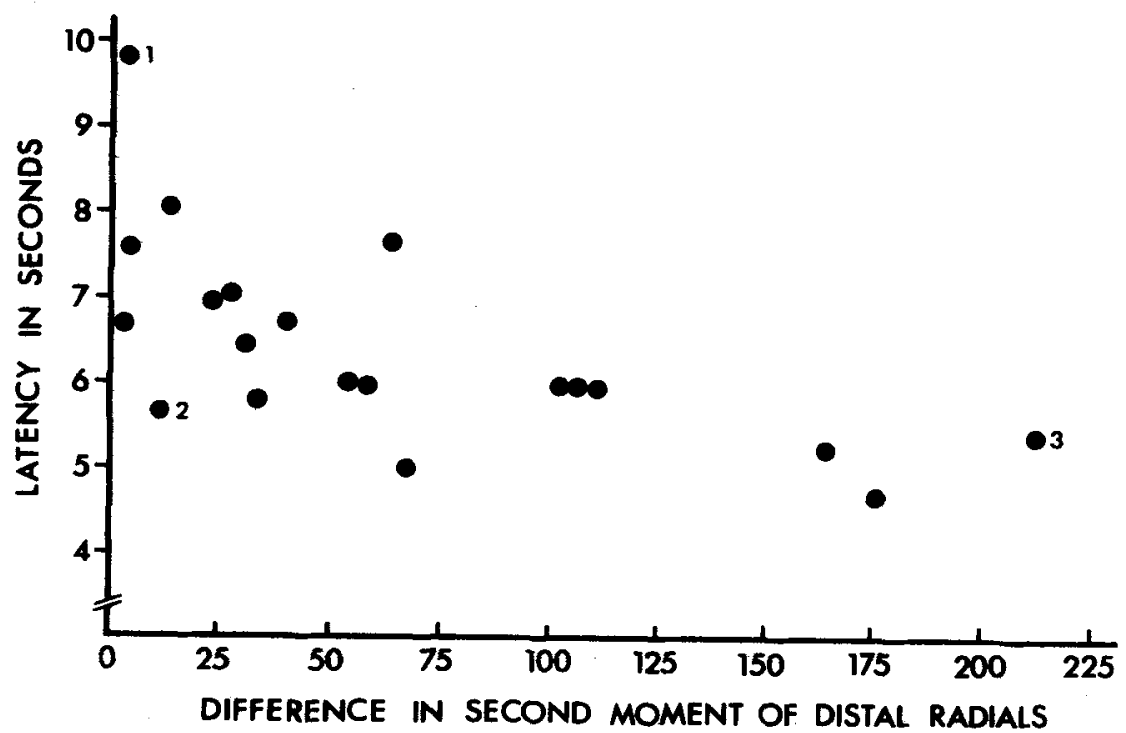

Problem 1 are similar in many ways, resulting in a difficult problem, while the Problem 3 forms differ greatly in dispersion of perimeter resulting in a fairly easy problem. The forms making up Problem 2 differ little in the physical measure graphed and yet the latency is nearly equal to that of Problem 3. However, because these forms differ a great deal in dispersion of area and perimeter along the vertical axis, Problem 2 is also easy.

\section{DISCUSSION}

Whether or not perceptual equivalence of the visual and tactual modalities has been demonstrated depends upon the level of comparison. Certainly, the relative difficulty of the various tasks as a function of number of sides demonstrates equivalence at the level of physical complexity. This result lends further support to Gibson's (1966) notion of partial equivalence of perceptual systems, as interpreted and extended by Owen and Brown (1970). If the visual latencies reflect primarily information processing time, then the tactual errors probably also reflect information processing capability, while the tactual latencies tend more to reflect information gaining. The decreasing correlation between tactual latencies and errors with increasing complexity supports this interpretation by suggesting that the two dependent variables are indices of different processes. In the cross-modal tasks, information from the visual form(s) may be used to limit the tactual search so that the matching latencies also reflect processing more than gaining time. When considered within side classes, however, the results reveal few instances of common information utilization. There is, to be sure, evidence of some common variance among the tasks, but the trends were not particularly instructive.

The major problem appears to be that there is no clear understanding of how the processes of pattern discrimination and pattern matching operate. Without that knowledge, it is somewhat presumptuous to begin comparing modalities and tasks. On the other hand, consideration of data gathered at the various levels of physical complexity-tactually, visually, and across modalities-by discrimination and matching, is more likely to lead to development of a model that will generalize across stimulus attributes, modalities, and tasks. Some understanding of the complications involved has been achieved.

The implication of the scattergraph pattern is relatively clear: The greater the difference in a salient feature, the more likely the latency will be short. If there is little or no difference along the dimension, another cue may be used instead, perhaps also resulting in a short latency. In fact, due to correlations among dimensions or to use of cues in combination, a short latency may be observed at any point on any dimension. As forms deviate less from the most compact form for a given sidedness level, they vary less and less along all dimensions, resulting in an increasing frequency of long latencies as the difference on any dimension decreases, i.e., there is generally less total information available for discrimination or matching. Therefore, while correlations of form measures with performance can give a descriptive indication of what information is useful, the relationship is neither linear nor bivariate normal, so that the predictive utility of linear correlation and regression depends upon the extent to which the assumptions are met.
Fig. 3. Scattergraph reflecting a correlation of -.62 between latency and difference in a physical measure for $\mathbf{2 0}$ problems in the VVT task at four sides.

It is evident from the results that multiple regression using factored dimensions to account for performance variability is not appropriate to construct a model isomorphic with the processes involved. In view of the efficacy of multiple regression in accounting for variability in judgments (Owen \& Brown, 1970 ) and for variation in recognizability of forms (Owen \& Andolsek, 1970), it should not be construed that the technique has little value. Rather, it appears that a different approach is required when dealing with how Ss detect differences and similarities in forms than in accounting for how a set of forms is arrayed from least to most by performance of some task. Accounting for the average discriminability of a form from all others in a set is not the same as accounting for the discrimination of that form from any particular form in the set. When $S$ is instructed to perform a task involving at least two forms as quickly and as accurately as possible, rather than being told to attend to certain parameters of the forms, he is free to use any stimulus relations available to correctly solve a problem. With the type of forms used, there is enough information available so that any problem may be solved in a number of ways. No consistency is required from one problem to the next, from one $S$ to the next, or from a given task to any other task. However, the high degree of similarity among $\mathrm{Ss}$ in the Owen and Andolsek (1970) recognition experiment and the high split-half

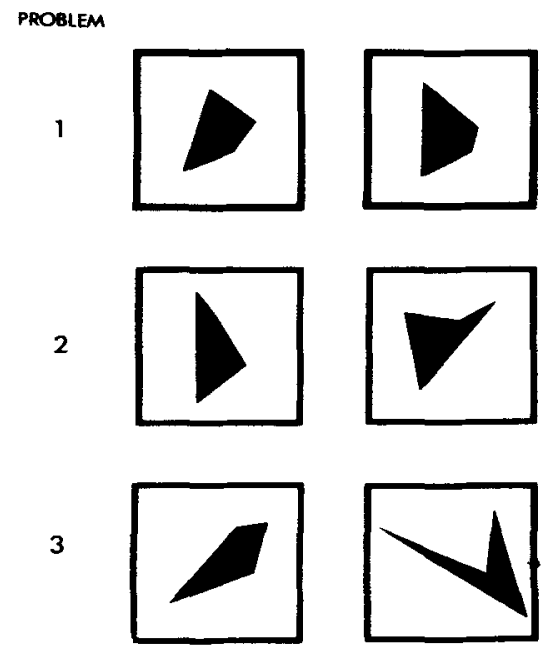

Fig. 4. Three form pairs corresponding to the problems numbered in Fig. 3. 
reliability found for two groups of $100 \mathrm{Ss}$ in the present visual-discrimination (VVV) task strongly suggest that there is inter-S consistency in problem solution. Consistency across modalities and across tasks has not been ": inpressive. Little is known, for example, about what $S$ does in oddity discrimination as opposed to what he does in solving a matching-to-sample problem. The oddity task is more likely to require assessing differences, while the matching task is more likely to require $S$ to attend to similarities. It is also possible that the two tasks may set $S$ to pick up different information, which by necessity limits the features available for comparisons occurring later in the perceptual processes. The problem is probably closely tied to the observation that latencies of "same" and "different" judgments are usually not equal (e.g., Bindra, Donderi, \& Nashisato, 1968; Hawkins, 1969; Nickerson, 1967).

The generality of the notion that $S$ has or develops a strategy that is applied to any discrimination problem needs critical examination. Where instructions, feedback, or other task demands do not suggest a strategy, information utilization might rather be determined by the relations available between forms making up a particular problem. The concept of an effective hierarchy of information utilization remains appropriate, since the saliency of features or properties will vary from problem to problem. Assuming that $S$ is capable of extracting the features needed to solve a discrimination or a matching problem, an adequate theory of what processing occurs must consider that incoming stimulation can carry the information for a comparison strategy. In other words, if task demands or previous problems do not suggest a strategy, the information in the problem will.

\section{REFERENCES}

ATTNEAVE, F., \& ARNOULT, M. D. The quantitative study of shape and pattern perception. Psychological Bulletin, 1956, 53, $452-471$.

BINDRA, D., DONDERI, D. C., \& NISHISATO, S. Decision latencies of "same" and "different" judgments. Perception \& Psychophysics, 1968, 3, 121-130.

BJÖRKMAN, M., GARVILL, J., \& MOLANDER, B. Cross-modal transfer as a function of preparatory set and distinctiveness of stimulus aspects. Reports from the Psychological Laboratories, University of Stockholm, 1965 , No. 186.

BOYNTON, R.' M., ELWORTH, C. L., MONTY, R. A., ONLEY, J. W., \& KLINGBERG, C. L. Overlap as a predictor of form discrimination under suprathreshold conditions. United States Air Force Air Research \& Development Technical Report, 1961, No. 61-99.

BROWN, D. R., \& BRUMAGHIM, S. H. Perceptual equivalence, pattern perception, and multidimensional methods. Perception \& Psychophysics, 1968, 4, 253-256.

BROWN, D. R., HITCHCOCK, JR., L., \& MICHELS, K. M. Quantitative studies in form perception: An evaluation of the role of selected stimulus parameters in the visual discrimination performance of human subjects. Perceptual \& Motor Skills, 1962, 14, 519-529.

BROWN, D. R., \& OWEN, D. H. The metrics of visual form: Methodological dyspepsia. Psychological Bulletin, 1967, 68, 243-259.

BRUMAGHIM, S. H., \& BROWN, D. R. Perceptual equivalence between visual and tactual pattern perception: An anchoring study. Perception \& Psychophysics, 1968, 4, 175-179.

CAVINESS, J. A., \& GIBSON, J. J. The equivalence of visual and tactual stimulation for the perception of solid forms. Paper presented at Eastern Psychological
Association, Atlantic City, April 1962.

CAVINESS, J. A., \& GIBSON, J. J. Crossmodal transfer and crossmodal discrimination of solid shapes. Paper presented at Eastern Psychological Association, Philadelphia, April 1964.

GAYDOS, H. F. Intersensory transfer in the discrimination of form. American Journal of Psychology, 1956, 69, 107-110.

GIBSON, J. J. The useful dimensions of sensitivity. American Psychologist, 1963, 18, 1-15.

GIBSON, J. J. The senses considered as perceptual systems. Boston: Houghton Mifflin, 1966.

HAWKINS, H. L. Parallel processing in complex visual discrimination. Perception \& Psychophysics, 1969, 5, 56-64.

LOBB, $\vec{H}$. Vision versus touch in form discrimination. Canadian Journal of Psychology, 1965, 19, 175-187.

NICKERSON, R. S. "Same"--"different" response times with multi-attribute stimulus differences. Perceptual \& Motor Skills, 1967, 24, 543-554. OWEN, D. H., \& BROWN, D. R. Visual and tactual form complexity: A psychophysical approach to perceptual equivalence. Perception \& Psychophysics, 1970, 7, 225-228.

OWEN, D. H., \& ANDOLSEK, M. A. Form recognition: Individual strategies in multivariate cue utilization. Perception \& Psychophysics, 1970, 7, 234-238.

PICK, A. D. Improvement of visual and tactual form discrimination. Journal of Experimental Psychology, 1965, 69, 331-339.

RUDEL, R. G., \& TEUBER, H. L. Crossmodal transfer of shape discrimination by children. Neuropsychologia, 1964, 2, 1-8. NOTES

1. This research was supported in part by Research Grant MH-12592 from the National Institute of Mental Health to the first author and in part by Research Grant HD-00909 from the National Institute of Child Health and Human Development to the second author.

2. Address: Department of Psychology; The Ohio State University, Columbus, Ohio, 43210.

(Accepted for publication August 26, 1969.) 